\section{Conserving uranium without the fast breeder}

The fast breeder, with all its dangers, is not the only way forward for the nuclear power programme. John Davies explains

NATURAL uranium, unaugmented by breeding, will provide a relatively small reserve of energy: uranium energy reserves amount to only onefifth those of oil. The best-promoted nuclear solutions to this problem are the fast breeder reactor (FBR) and controlled fusion. The former is a means of stretching the uranium resources by two orders of magnitude; the latter promises virtually unlimited power from sea-water. Neither method is straightforward, although both were being discussed in undergraduate lectures twenty years ago. A commercial FBR is not yet operational and the design of a fusion reactor providing energy break-even is still in the unplanned future. Moreover FBRs, with their use of plutonium, are responsible for many of the strong, reasoned objections to nuclear power. However, few have realised that there are several other ways of breeding fissile materials that avoid fast reactors and their problems.

Breeding-creating fissile from nonfissile material-requires an intense source of neutrons. Neutrons can be produced by an FBR; or by a fusion reactor (yet to be built); or by using proton accelerators. The last option has not been widely discussed.

To make the arguments clear, I shall first describe some of the basic physics occurring in reactors, particularly to explain what "breeding" is and why it is essential. I shall then look at the objections to FBRs before examining the alternative means of breeding to demonstrate a system that I believe is practical, economically viable, and safe.

As medium-weight nuclei are the most stable, considerable amounts of energy are generated either when two light nuclei fuse together, or when a heavy nucleus splits into two smaller ones (undergoes fission). To exploit either possibility requires an energy barrier to be overcome. (If there were no energy barrier all light or heavy nuclei would by now have fused or fissioned leaving only medium-weight nuclei.) Another requirement (in fission) is a mechanism for maintaining a chain reaction-where the fission of one nucleus in turn induces the fission of others.

In fission, the energy barrier is, roughly speaking, the surface tension of the nuclear droplet. A heavy nucleus approaching fission becomes progress- ively distorted, and the energy of the increased surface provides the fission barrier (small droplets of water are stable for the same reason). The impact and interaction of a neutron of the right energy overcomes this energy barrier. If, in turn, each resulting fission releases more neutrons (as it will, as heavy nuclei are neutron-rich) the neutrons will maintain a chain reaction with an unending succession of nuclei being induced to split.

Let us now consider fission in more detail. Thermal reactors use the fissile nuclei ${ }^{233} \mathrm{U},{ }^{235} \mathrm{U}$ and ${ }^{239} \mathrm{Pu}$ where the binding energy provided by the capture of a thermal neutron is sufficient to overcome the surface energy barrier against prompt fission of the resulting ${ }^{234} \mathrm{U},{ }^{236} \mathrm{U}$ and ${ }^{240} \mathrm{Pu}$. Each induced fission also provides several neutrons which can then start a chain reaction. But the trouble is that the isotopes ${ }^{233} \mathrm{U}$ and ${ }^{239} \mathrm{Pu}$ are artificial; and ${ }^{235} \mathrm{U}$ is a rare isotope of uranium, the most abundant being ${ }^{238} \mathrm{U}$.

Unfortunately, the abundant ${ }^{238} \mathrm{U}$ will not do for fission, as the figure shows. This plots the effective number of neutrons per fission $(\eta)$ against the kinetic energy of the captured neutron; and shows the fission neutron energy snectra in thermal and fast reactors. In contrast with (say) ${ }^{236} \mathrm{U}$, the last, odd neutron in ${ }^{239} \mathrm{U}$ has a smaller binding energy. Consequently only the most energetic neutron coming from fission can cause further fission by capture in ${ }^{238} \mathrm{U}$ and so ${ }^{238} \mathrm{U}$ cannot maintain a chain reaction.

With thermal reactors only, using natural uranium, uranium resources are insufficient to maintain a long-term nuclear energy programme. The only naturally occurring fissile isotope is ${ }^{235} \mathrm{U}$, present at 1 part in 140 of ${ }^{239} \mathrm{U}$. Moreover it is only part-burnt in thermal reactors. This leads to the idea of trying to "breed" fissile isotopes, by using the excess neutrons in the chain reaction to create fissile nuclei in otherwise non-fissile elements.

From each fission of ${ }^{235} \mathrm{U}$ one neutron is required to maintain the chain reaction and 0.2 neutrons escape. Any excess over 1.2 neutrons could be used to convert ${ }^{238} \mathrm{U}$ or ${ }^{232} \mathrm{Th}$ to fissile ${ }^{239} \mathrm{Pu}$ or ${ }^{233} \mathrm{U}$ via

$$
{ }^{238} \mathrm{U}(\mathrm{n}, \gamma){ }^{239} \mathrm{U} \underset{23 \mathrm{~min}}{\stackrel{\beta-}{\longrightarrow}}{ }^{239} \mathrm{~Np} \underset{23 \text { days }}{\stackrel{\beta-}{\longrightarrow}}{ }^{239} \mathrm{Pu}
$$

${ }^{232} \mathrm{Th}(\mathrm{n}, \gamma){ }^{233} \mathrm{Th} \underset{23 \mathrm{~min}}{\longrightarrow}{ }^{233} \mathrm{~Pa} \underset{27 \text { days }}{\longrightarrow}{ }^{233} \mathrm{U}$

(Here the notation $\mathrm{A}(\mathrm{n}, \gamma) \mathrm{B}$ means nucleus $\mathrm{A}$ absorbs an incoming neutron and becomes nucleus $\mathrm{B}$, emitting a gamma ray (a photon) in the process. The notation $\mathrm{B} \underset{x \min }{\stackrel{\beta}{\longrightarrow}} \mathrm{C}$ means $\mathrm{B}$ decays by $\beta^{-}$emission to $C$ with a half-life of $x$ minutes.)

The difference between the two reactions is that the first needs fast neutrons, while the second can use slow ones. The reaction ${ }^{238} \mathrm{U} \rightarrow{ }^{238} \mathrm{Pu}$ occurs to a small extent in present thermal reactors (in so far as there are a few fast neutrons around) and chemical processing can enable up to $1 \%$ of all available uranium to be burnt. Even so there is probably little point in planning further thermal reactors (as there will be insufficient uranium to fuel them and existing reactors).

\section{Breeding in a fertile blanket}

The fissile material for reactor fuel can be produced from fertile material using the excess neutrons from a reactor and the above reactions. In practice this could be done by surrounding the reactor with a 'blanket' of fertile material where the spare neutron would then 'breed' new fuel. In fact if $\eta \geqslant 2.2$, with one more neutron per fission than required to maintain the chain reaction, then breeding in a fertile blanket will maintain or increase the fissile content and allow almost all the uranium to be burnt. The figure shows that a fast reactor burning ${ }^{239} \mathrm{Pu}$ surrounded by a fertile ${ }^{238} \mathrm{U}$ blanket absorbing the excess fast neutrons is the best bet.

Is this really true? Much of the reasoned objections to nuclear power are consequent on the following problems of the fast breeder reactor:

- New technology-breeding around a $500 \mathrm{MW}$ dustbin whose reactivityburning rate-can be accidentally increased in micro-seconds; no artificial control is fast enough.

- Time-whether thermal reactors can produce enough ${ }^{239} \mathrm{Pu}$ to fire a FBR regime whose time to breed the initial fissile content is anyway commensurate with their working life.

- The expensive, messy and dangerous chemical separation of fissile materials, in particular ${ }^{239} \mathrm{Pu}$, from spent fuel elements. This separation greatly increases the possibility of

- ${ }^{239} \mathrm{Pu}$ being diverted into nuclear weapons; this is further exacerbated by the considerable transporting of fissile material and by inaccuracies of assay of ${ }^{239} \mathrm{Pu}$, which can be so large as to be greater than the amounts needed for 
many bombs. (This is called the "Muf"-materials unaccounted for-. problem.)

- The cost-as yet not evaluatedcapital, running and political-mespecially for trying to make them safe.

Thermal reactors on the other hand involve established technology. The figure shows that such a reactor fuelled with ${ }^{233} \mathrm{U}$ has a breeding gain $(\eta-2.2)$ greater than 1.00 i.e., as much ${ }^{233} \mathrm{U}$ will be created in a ${ }^{232} \mathrm{Th}$ blanket (absorbing the slow neutrons) as is burnt as fuel. Unlike ${ }^{239} \mathrm{Pu},{ }^{233} \mathrm{U}$ is automatically safeguarded against illegal diversion into bomb-making by the intense $\gamma$-rays coming from the simultaneously produced ${ }^{232} \mathrm{U}$ : additionally one can add sufficient ${ }^{238} \mathrm{U}$ to make life difficult for the bomb maker but still keeping the ${ }^{233} U$ fissile. Such reactors avoid plutonium and the technological problems of FBRs. Also they ease the waste problem: the proportion of difficult transuranic elements (which take a long time to decay) is considerably reduced.

Thermal reactors fuelled on ${ }^{233} \mathrm{U}$ can have a breeding gain of 1.00 or greater in a Th blanket. But considerable development would be necessary to make this economic. Additionally chemical re-processing of the spent fuel rods, as yet undeveloped, may be necessary.

Consider what a well established, practical and economic reactor can do with a Th-U cycle. The Canadian Deuterium Uranium (CANDU) series of heavy water moderated thermal reactors have high neutron economy and use natural uranium so there is no chemical separation. They can operate on a Th-U cycle with breeding gain as large as 0.92 . Thus to use all the available thorium requires only $8 \%$ of the ${ }^{232} \mathrm{Th}$ atoms to be converted to ${ }^{233} \mathrm{U}$ by non-nuclear means.

Some fusion enthusiasts are lowering their expensive sights, ignoring energy output and promoting fusion reactors as intense neutron sources for fertile to fission conversion. This is not surprising! Fusion reactor design is replete with problems such that only a 2000 MW TOKAMAK has promise of creating as much energy as it consumes even with the most easily ignited $D(T, n)$ ${ }^{4} \mathrm{He}$ reaction. It is possible that the 14 $\mathrm{MeV}$ neutron is far more valuable for breeding than for heating, each neutron converting two or more fertile atoms into fissile ones. Even so despite the relaxed conditions for only providing neutrons fusion technology is still a dream and many regard a hybrid arrangement as combining the worst features of fission and fusion.

\section{Proton accelerators}

Of all the big advanced technologies, that of accelerator building can claim to have established the most consistent track record. The high intensity accelerator at Los Alamos is well on the way to its design beam current, the CERN SPS is working ahead of schedule within its design budget while the high energy accelerator at the Fermi Laboratory is already $2 \frac{1}{2}$ times its original design energy. At several laboratories high intensity accelerators are now replacing fission reactors as research instruments providing high intensity neutrons.

The intense neutron fluxes from such particle accelerators can effect fertile-to-fissile conversion and so produce far more energy than they consume. A $1 \mathrm{GeV}$ proton interacting in a uranium target produces 60 low energy neutrons by a process known as spallation; each neutron can change one ${ }^{232} \mathrm{Th}$ atom into one ${ }^{233} \mathrm{U}$ atom in a fertile thorium blanket surrounding the uranium target; each ${ }^{233} \mathrm{U}$ atom can fission in a CANDU reactor producing $200 \mathrm{MeV}$ of available kinetic energy; of every $100{ }^{233} \mathrm{U}$ atoms so burnt, 92 will have been produced in a thorium blanket around the same or similar reactor while only 8 will have had to be produced via an accelerator. So effectively a single $1000 \mathrm{MeV}$ proton produces

$$
60 \times \frac{100}{8} \times 200=150,000 \mathrm{MeV}
$$

This is possibly a lower estimate as there may be neutron multiplication in the blanket.

Accelerators are inherently cheaper to build than reactors and a large proportion of the accelerator operating power could be provided from heat generated in the target. The accelerator design criteria are high efficiency of radio-frequency power to beam energy conversion, minimal power usage in other parts of the accelerator (such as magnets) and high extraction efficiency.

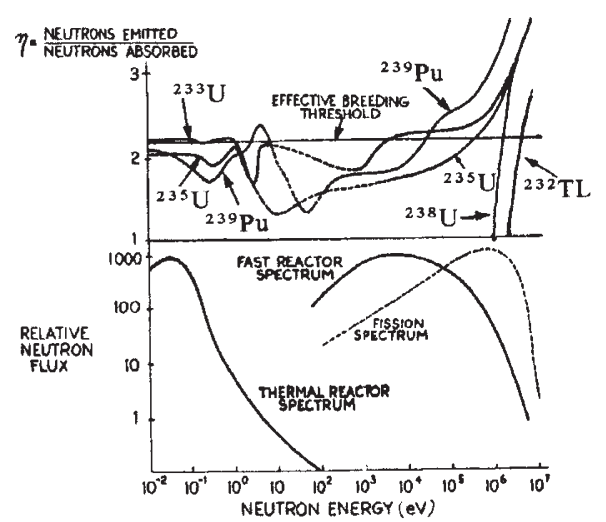

The ratio of neutrons emitted to neutrons absorbed, in collisions between neutrons and nuclei, plotted against neutron energy. Also shown are the neutron spectra from fission directly (dotted line) and in a thermal and fast breeder reactor
Most work on meeting these requirements has involved a high intensity, medium energy, linear accelerator which is essentially an extension of the $800 \mathrm{MeV}$ Los Alamos machine, now being worked up to $1 \mathrm{~mA}$ beam current. However, as a good example of lateral thinking, is a suggestion that these requirements can also be met by a low current, very high energy accelerator, an extension of the 400 GeV CERN SPS or the $500 \mathrm{GeV}$ accelerator at the Fermilab.

A Canadian group including Fraser, Hoffman and Tunnicliffe at Chalk River are designing a $100-300 \mathrm{~mA}$, $1 \mathrm{GeV}$ proton linear accelerator that would breed enough fuel to supply the $8 \%$ deficiencies of a dozen, $1,000 \mathrm{MW}$ CANDU reactors operating on a uranium-thorium cycle. The fertile blanket would consist of new fuel pins that would subsequently go directly to the reactor after a pause to allow for ${ }^{233} \mathrm{~Pa}$ decay. A sophisticated fuel management scheme is conceivable in which partially-burned fuel pins are upgraded by re-jnsertion in the target and then returned to the reactor.

Another suggestion, from Wilson at Fermilab, is for a superconducting $1000 \mathrm{GeV}$ accelerator with an intensity of $1-2 \times 10^{13}$ protons per second. At this energy the number of spallation neutrons is still increasing linearly with energy and so a single proton will produce 60,000 neutrons in a block of uranium. High energies and low currents mean low beam loss and efficient conversion of radio-frequency power to beam energy for protons travelling close to the velocity of light. Fermilab's superconducting energy doubler will provide $1,000 \mathrm{GeV}$ protons. The intensity required for energy break-even must be increased from the present $10^{12}$ protons per pulse by an amount that one has come to expect over an accelerator's life-time. This neglects the effective $\times \frac{100}{8}$ energy

multiplication obtained by breeding ${ }^{233} \mathrm{U}$ for CANDU reactors.

Accelerator spallation neutron sources are being built at several laboratories as research instruments to replace fission reactors as a source of high intensity neutrons. Accelerator scientists regard upgrading spallation sources for breeding purposes as a reasonable extrapolation of existing, successful techniques. This would make the thorium-uranium cycle in thermal reactors a serious contender as a nuclear fuel source with the added bonus (to many the over-riding attraction) that objectionable material need never be removed from the fuel rods. 